\title{
Sex-specific hemodynamic and non-hemodynamic determinants of aortic root size in hypertensive subjects with left ventricular hypertrophy
}

\author{
José AA Cipolli ${ }^{1,3}$, Felipe AS Souza ${ }^{1,3}$, Maria CS Ferreira-Sae ${ }^{1}$, José A Pio-Magalhães ${ }^{1}$, Eugênio S Figueiredo ${ }^{2}$,
} Vanessa G Vidotti ${ }^{2}$, José R Matos-Souza ${ }^{1}$, Kleber G Franchini ${ }^{1}$ and Wilson Nadruz Jr$^{1}$

Aortic root (AoR) dilatation is more frequently observed in hypertensive individuals and is independently associated with left ventricular (LV) hypertrophy. Although the LV structure has sex-specific predictors, it remains unknown whether there are gender-related differences in the determinants of AoR size. We carried out a cross-sectional analysis of clinical, laboratory, anthropometric, funduscopic and echocardiographic features of 438 hypertensive patients with LV hypertrophy (266 women and 172 men). Women with enlarged AoR had higher cardiac output $(P=0.0004)$, decreased peripheral vascular resistance $(P=0.009)$, higher prevalence of mild aortic regurgitation $(P=0.02)$ and increased waist circumference $(P=0.04)$, whereas AoR-dilated men presented with a higher prevalence of concentric LV hypertrophy $(P=0.0008)$ and mild aortic regurgitation $(P=0.005)$ and increased log $C$-reactive protein levels $(P=0.02)$, compared with sex-matched normal AoR subjects. In women, AoR dilatation associated with cardiac output, mild aortic regurgitation and waist circumference in a multivariate model including age, body surface area, height, homeostasis model assessment index, LV mass index, diastolic blood pressure, menopause status and use of antihypertensive medications as independent variables. Conversely, AoR dilatation associated with LV relative wall thickness, log C-reactive protein and mild aortic regurgitation without contributions from diastolic blood pressure, height, body surface area, LV mass index, peripheral vascular resistance and antihypertensive medications in men. Taken together, these results suggest that both volume overload and abdominal obesity are related to AoR dilatation in hypertensive women, whereas AoR enlargement is associated more with inflammatory and myocardial growth-related parameters in hypertensive men with LV hypertrophy.

Hypertension Research (2009) 32, 956-961; doi:10.1038/hr.2009.134; published online 28 August 2009

Keywords: aortic root; gender; left ventricular hypertrophy; volume overload

\section{INTRODUCTION}

Dilatation of the aortic root (AoR) is frequently associated with higher cardiovascular mortality. ${ }^{1,2}$ Therefore, increased importance has been devoted to the study of the biological determinants of AoR size. AoR dilatation is observed more frequently in hypertensive than in normotensive individuals $s^{3,4}$ and its prevalence is higher in patients with complicated hypertension, ${ }^{5-7}$ supporting the idea that it is a targetorgan damage marker. However, this assumption has been challenged by several lines of evidence showing no difference in AoR diameter between hypertensive and normotensive individuals after adjusting for age and body size. ${ }^{3,4,8}$

Left ventricular (LV) hypertrophy is a major predictor of AoR dilatation in hypertensive subjects, independent of age, body size and gender. ${ }^{6,7}$ This observation argues against the notion that changes in AoR diameter are solely explained by variations in body size and age, and points toward AoR and LV remodeling as processes with common pathophysiological mechanisms. In addition, it suggests that hypertensive patients with LV hypertrophy comprise a population with attractive features for the study of potential determinants of AoR size.

Mounting evidence showed that there are gender-related differences in the predictors of LV structure in hypertensive subjects. In this regard, LV mass and geometry show sex-specific relationships with body mass composition, metabolic factors and other markers of target-organ damage. ${ }^{9-12}$ On the other hand, AoR size and the prevalence of AoR dilatation are clearly higher in men, ${ }^{4-6}$ indicating that gender has a major role in the regulation of the AoR phenotype. Nevertheless, it remains unknown whether there are sex-related differences in the determinants of AoR diameter in hypertensive subjects. Therefore, the aim of this study was to evaluate the impact

${ }^{1}$ Department of Internal Medicine, School of Medicine, University of Campinas, Campinas, Brazil and ${ }^{2}$ Department of Ophthalmology, School of Medicine, University of Campinas, Campinas, Brazil

3These authors contributed equally to this work.

Correspondence: Dr W Nadruz Junior, Departamento de Clínica Médica, Faculdade de Ciências Médicas, University of Campinas, Universidade Estadual de Campinas, Cidade Universitária Zeferino Vaz, Campinas, SP 13081-970, Brazil.

E-mail: wilnj@fcm.unicamp.br

Received 7 June 2009; revised 7 July 2009; accepted 9 July 2009; published online 28 August 2009 
of gender on the determinants of AoR size in a sample of patients with hypertension and LV hypertrophy.

\section{METHODS}

A total of 438 consecutive patients ( 325 Whites and 113 Blacks), followed up at the Hypertension Unit of the University of Campinas, were included in the study. Inclusion criteria were as follows: (1) hypertension, defined as systolic blood pressure $\geqslant 140 \mathrm{~mm} \mathrm{Hg}$, or diastolic blood pressure $\geqslant 90 \mathrm{~mm} \mathrm{Hg}$ or current antihypertensive medication use; (2) echocardiographic diagnosis of LV hypertrophy; and (3) age over 18 years and no evidence of moderate or severe cardiac valve disease, hypertrophic cardiomyopathy, previous myocardial infarction, neoplastic disease and suspected secondary hypertension. The studied patients underwent a cross-sectional analysis of the following diagnostic procedures: (1) clinical blood pressure measurements, (2) anthropometric measurements, (3) routine investigations (blood chemistry, creatinine clearance and urinary albumin-creatinine ratio analyses), (4) funduscopic examination and (5) an echocardiogram. The study was approved by the ethics committee of the University of Campinas, and written consent was obtained from all participants.

Blood pressure was measured using a validated digital oscillometric device (Omron HEM-705CP, Omron Healthcare, Kyoto, Japan) with appropriate cuff sizes. ${ }^{10}$ Body mass index was calculated as body weight divided by height squared (expressed in $\mathrm{kg} \mathrm{m}^{-2}$ ). Waist circumference was measured at the midpoint between the lowest rib and iliac crest and hip circumference at the level of the greater trochanters.

Blood samples were obtained in the morning after $12 \mathrm{~h}$ of fasting for analysis of total cholesterol, cholesterol fractions, glucose, insulin and C-reactive protein levels. The homeostasis model assessment index was calculated as follows: glucose $(\mathrm{mg}$ per $100 \mathrm{ml}) \times$ insulin $\left(\mu \mathrm{U} \mathrm{ml}^{-1}\right) / 405 .{ }^{10}$ Funduscopic diagnosis of hypertensive retinopathy was evaluated bilaterally by a skilled ophthalmologist, who had no knowledge of the patient's clinical characteristics. The funduscopic findings were graded using the Keith-Wagener classification. Albuminuria was evaluated in patients by measuring the albumin-creatinine ratio in morning urine samples. In addition, creatinine clearance was also measured. Diabetes mellitus was diagnosed if fasting blood glucose was $\geqslant 126 \mathrm{mg}$ per $100 \mathrm{ml}$ or when participants were taking hypoglycemic medications, whereas metabolic syndrome was defined in accordance with the National Cholesterol Education Program Panel III criteria. ${ }^{13}$ Women with reported amenorrhea for more than 12 months, except for pregnancy, were identified as postmenopausal.

Echocardiography studies were conducted on each subject at rest in the left lateral decubitus position using a Vivid 3 Pro (General Electric, Milwaukee, WI, USA) apparatus equipped with a $2.5-\mathrm{MHz}$ transducer as described previously. ${ }^{14}$ AoR diameter, LV end-diastolic and end-systolic diameters, interventricular septum thickness, posterior wall thickness and LV mass were measured in accordance with the American Society of Echocardiography guidelines. ${ }^{15}$ AoR diameter was measured at the level of Valsalva's sinuses by M-mode tracings, under two-dimensional control, as the maximal distance between the two leading edges of the anterior and posterior AoR walls at the end diastole. Relative wall thickness was computed as twice the posterior wall thickness divided by the LV end-diastolic diameter. Mild aortic regurgitation was identified on the basis of the extent of diastolic turbulent flow in the LV, indicated by a variance signal, by a jet occupying $<20 \%$ of the aortic annular diameter at its origin and extending less than halfway to the tip of the anterior mitral leaflet. ${ }^{16}$ All the recordings were made by the same physician, who was unaware of other data relating to the subjects. The reproducibility of both acquiring and measuring LV mass and AoR size was determined in recordings obtained from 10 subjects. Intraobserver LV mass and AoR size variabilities were $<8$ and $<3 \%$, respectively, whereas interobserver variabilities of these parameters were $<11$ and $<5 \%$, respectively.

Systolic volume was generated from Doppler interrogation of transaortic flow at the aortic annular level and aortic valve cross-sectional area. ${ }^{17}$ Cardiac output was calculated as systolic volume $\times$ cardiac frequency, whereas peripheral vascular resistance was obtained using the formula: mean blood pressure/ cardiac output.

Aortic root was considered dilated when its diameter was $\geqslant 37 \mathrm{~mm}$ in women and $40 \mathrm{~mm}$ in men. These cutoff points were the 98 th percentile $(>2$ s.d.) values ${ }^{7}$ in a group of 356 normotensive, non-obese (body mass index between 20 and $25 \mathrm{~kg} \mathrm{~m}^{-2}$ ) and apparently normal adults (196 women and 160 men; mean age: $56.0 \pm 0.6$ years) evaluated in an outpatient unit for a cardiovascular checkup in the previous 3 years. LV hypertrophy was defined with the use of cutoff point LV mass/height ${ }^{2.7}>51$ for both genders, ${ }^{18}$ whereas concentric geometry was considered if the relative wall thickness was $\geqslant 0.45$. ${ }^{19}$

Descriptive statistical results are expressed as mean \pm s.e. Chi-squared test was used to compare categorical variables, whereas the unpaired $t$-test and Mann-Whitney test compared the parametric and nonparametric continuous variables, respectively. Pearson's or Spearman's method was used to assess univariate correlations between clinical features and AoR size. Stepwise and logistic regression analyses evaluated the independent determinants of AoR diameter. A $P$-value $<0.05$ was considered significant.

\section{RESULTS}

\section{Gender-specific features of hypertrophic patients according to AoR} dilatation

Aortic root dilatation, defined by the sex-specific criteria of $40 \mathrm{~mm}$ in men and $37 \mathrm{~mm}$ in women, was found in 46 of 438 patients, with an overall prevalence rate of $10.5 \%$. A dilated AoR was present in $15.1 \%$ of men and in $7.5 \%$ of women. The clinical features of the studied subjects are presented in Table 1. Women and men with AoR enlargement were older than those with normal AoR size, whereas larger waist circumference and higher prevalence of postmenopause were found in females with dilated AoR compared with normal AoR women. Conversely, men with AoR dilatation showed higher log C-reactive protein levels compared with those with normal AoR size. Body surface area, body mass index, hip circumference, homeostasis model assessment index and use of antihypertensive medications were similar in both genders according to AoR size. In addition, subjects with AoR dilatation showed no differences in lipid fractions and in the prevalence of smoking and diabetes mellitus (Supplementary data).

Table 2 shows the echocardiographic features of men and women, as well as their statistical comparisons according to the presence or absence of AoR dilatation. Men with AoR dilatation showed increased LV wall thickness, relative wall thickness, LV mass index and a higher prevalence of LV concentric hypertrophy, whereas women with enlarged AoR displayed increased LV end-diastolic diameter compared with sex-matched subjects with normal AoR size. Moreover, subjects with AoR dilatation of both genders showed increased prevalence of mild aortic regurgitation. In contrast to LV structural parameters, no variations in retinal vascular changes, as well as in urinary albumincreatinine ratio and creatinine clearance, were found in both genders according to the presence or absence of AoR enlargement (Supplementary data).

The hemodynamic features of studied subjects are shown in Table 3. Women with enlarged AoR showed higher stroke volume and cardiac output in addition to lower peripheral vascular resistance than did those with normal AoR size. On the other hand, no differences in hemodynamic parameters were found in men according to AoR size.

\section{Univariate correlations between AoR size and studied variables}

To determine the sex-specific correlates of AoR size, univariate analyses were carried out (Table 4). In hypertensive women, AoR size showed a positive and major correlation with LV end-diastolic diameter, waist circumference, LV mass index, stroke volume and cardiac output and displayed a weaker direct relationship with age, body surface area, hip circumference, body mass index, LV wall thickness, postmenopause, mild aortic regurgitation and height. 
Table 1 Clinical characteristics of subjects with aortic root dilatation

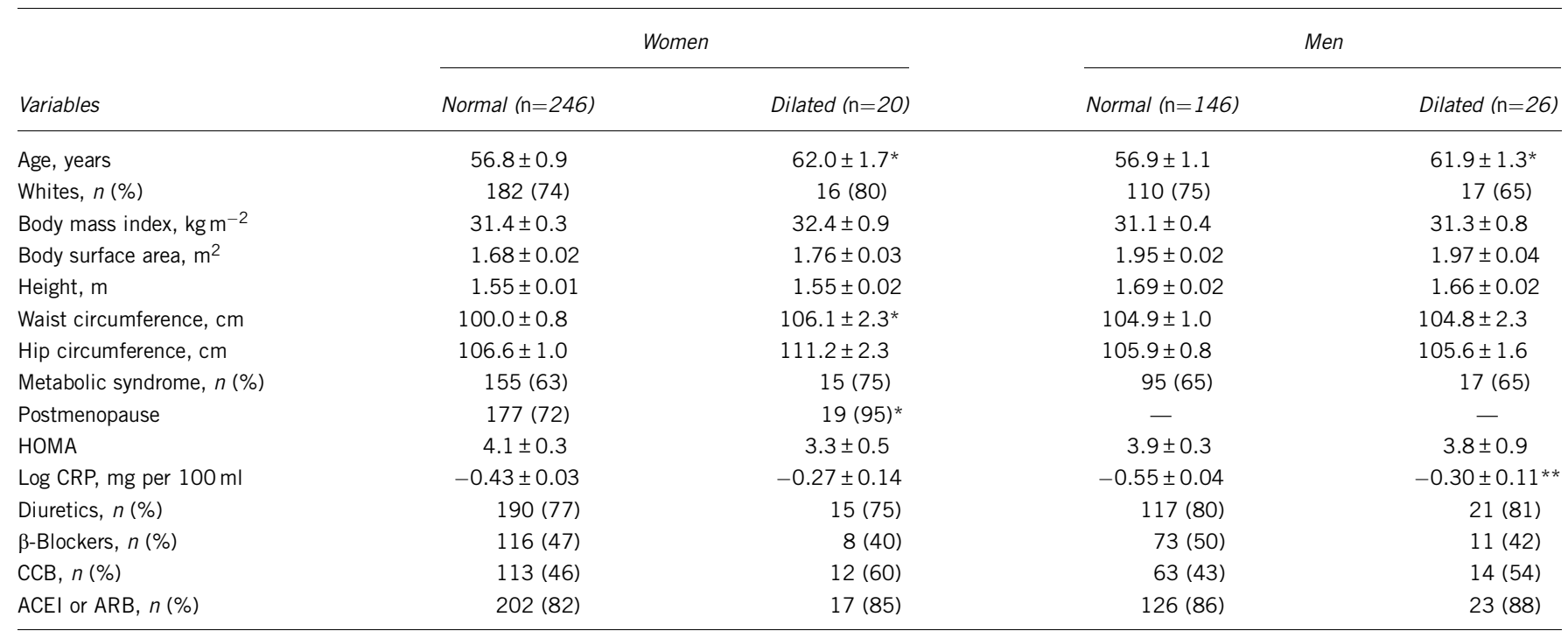

Abbreviations: ACEI, angiotensin-converting enzyme inhibitor; AoR, aortic root; ARB, angiotensin receptor blocker; CCB, calcium channel blocker; CRP, C-reactive protein; HOMA, homeostasis model assessment index.

${ }^{*} P=0.04$ and ${ }^{*} P=0.02$ compared with sex-matched subjects with normal AoR size.

Table 2 Echocardiographic features of subjects with aortic root dilatation

\begin{tabular}{|c|c|c|c|c|c|c|}
\hline \multirow[b]{2}{*}{ Variables } & \multicolumn{3}{|c|}{ Women } & \multicolumn{3}{|c|}{ Men } \\
\hline & Normal $(\mathrm{n}=246)$ & Dilated $(\mathrm{n}=20)$ & P-value & Normal $(\mathrm{n}=146)$ & Dilated $(\mathrm{n}=26)$ & P-value \\
\hline Aorta, mm & $31.2 \pm 0.2$ & $38.7 \pm 0.5$ & $<0.0001$ & $34.2 \pm 0.2$ & $42.0 \pm 0.4$ & $<0.0001$ \\
\hline LV end-diastolic diameter, $\mathrm{mm}$ & $48.8 \pm 0.3$ & $52.3 \pm 1.3$ & 0.002 & $53.0 \pm 0.5$ & $53.1 \pm 1.0$ & NS \\
\hline Posterior wall thickness, mm & $11.0 \pm 0.1$ & $10.9 \pm 0.3$ & NS & $11.5 \pm 0.1$ & $12.6 \pm 0.2$ & 0.0002 \\
\hline Interventricular septum, mm & $11.1 \pm 0.1$ & $11.2 \pm 0.4$ & NS & $11.6 \pm 0.1$ & $12.9 \pm 0.2$ & $<0.0001$ \\
\hline Relative wall thickness & $0.45 \pm 0.01$ & $0.42 \pm 0.02$ & NS & $0.44 \pm 0.01$ & $0.47 \pm 0.01$ & 0.009 \\
\hline LV concentric hypertrophy, $n(\%)$ & $108(44)$ & $5(25)$ & NS & $55(38)$ & $19(73)$ & 0.0008 \\
\hline LV mass index, $\mathrm{g} / \mathrm{h}^{2.7}$ & $79.8 \pm 1.5$ & $86.8 \pm 4.5$ & NS & $78.6 \pm 1.7$ & $88.4 \pm 2.8$ & 0.02 \\
\hline Ejection fraction, \% & $66.2 \pm 0.4$ & $65.3 \pm 2.1$ & NS & $62.6 \pm 0.8$ & $62.4 \pm 1.5$ & NS \\
\hline Mild aortic regurgitation, $n(\%)$ & $45(18)$ & $8(40)$ & 0.02 & $30(21)$ & $12(46)$ & 0.005 \\
\hline
\end{tabular}

Abbreviations: LV, left ventricular; NS, nonsignificant.

Table 3 Hemodynamic features of subjects with aortic root dilatation

\begin{tabular}{|c|c|c|c|c|c|c|}
\hline \multirow[b]{2}{*}{ Variables } & \multicolumn{3}{|c|}{ Women } & \multicolumn{3}{|c|}{ Men } \\
\hline & Normal $(n=246)$ & Dilated $(\mathrm{n}=20)$ & $\mathrm{P}$-value & Normal $(\mathrm{n}=146)$ & Dilated $(n=26)$ & $\mathrm{P}$-value \\
\hline Systolic blood pressure, $\mathrm{mm} \mathrm{Hg}$ & $152.1 \pm 1.9$ & $143.6 \pm 4.5$ & NS & $149.7 \pm 2.1$ & $152.5 \pm 5.0$ & NS \\
\hline Diastolic blood pressure, $\mathrm{mm} \mathrm{Hg}$ & $89.1 \pm 1.1$ & $84.1 \pm 3.3$ & NS & $88.4 \pm 1.2$ & $88.7 \pm 2.5$ & NS \\
\hline Heart rate, b.p.m. & $65.1 \pm 0.4$ & $67.3 \pm 1.4$ & NS & $64.5 \pm 0.6$ & $63.3 \pm 1.6$ & NS \\
\hline Stroke volume, ml & $82.3 \pm 1.2$ & $95.5 \pm 6.0$ & 0.003 & $92.9 \pm 1.8$ & $93.6 \pm 3.7$ & NS \\
\hline Cardiac output, I per min & $5.34 \pm 0.08$ & $6.33 \pm 0.32$ & 0.0004 & $5.97 \pm 0.12$ & $5.92 \pm 0.26$ & NS \\
\hline Peripheral vascular resistance, dynes $\sec \mathrm{cm}^{-5}$ & $1716 \pm 38$ & $1381 \pm 77$ & 0.009 & $1549 \pm 40$ & $1594 \pm 121$ & NS \\
\hline
\end{tabular}

Abbreviations: b.p.m., beats per minute; NS, nonsignificant.

Conversely, AoR size correlated inversely with the peripheral vascular resistance and ejection fraction in this gender. In men, AoR diameter correlated directly with the LV wall thickness, LV mass index, mild aortic regurgitation, relative wall thickness, age, log C-reactive protein and peripheral vascular resistance. Finally, no significant relationship was observed between AoR size and creatinine clearance, log urinary albumin-creatinine, retinal vascular changes, lipid fractions, smoking, diabetes mellitus or antihypertensive medications in both genders (data not shown).

\section{Multivariate determinants of AoR size and dilatation}

Stepwise and logistic regression analyses were carried out to evaluate the independent contributions of different factors to AoR size and AoR dilatation, respectively. In women, AoR size associated with 
Table 4 Univariate correlation analysis of aortic root size

\begin{tabular}{|c|c|c|}
\hline Variables & Women $(n=266)$ & Men $(\mathrm{n}=172)$ \\
\hline Age & $0.16^{*}$ & $0.22^{*}$ \\
\hline Body surface area & $0.22 * *$ & -0.02 \\
\hline Body mass index & $0.17^{*}$ & 0.01 \\
\hline Height & $0.14^{* * *}$ & -0.02 \\
\hline Waist circumference & $0.29 * *$ & 0.06 \\
\hline Hip circumference & $0.22 * *$ & 0.03 \\
\hline Systolic blood pressure & -0.06 & 0.02 \\
\hline Diastolic blood pressure & -0.06 & 0.08 \\
\hline Metabolic syndrome & 0.07 & 0.08 \\
\hline Postmenopause & $0.14^{* * *}$ & - \\
\hline Homeostasis model assessment index & -0.03 & -0.02 \\
\hline Log C-reactive protein & 0.04 & $0.16^{* * *}$ \\
\hline Left ventricular end-diastolic diameter & $0.35^{* *}$ & 0.02 \\
\hline Posterior wall thickness & $0.15^{* * *}$ & $0.35^{* *}$ \\
\hline Interventricular septum & $0.15^{* * *}$ & $0.38 * *$ \\
\hline Relative wall thickness & -0.07 & $0.26^{* *}$ \\
\hline Left ventricular mass index & $0.27^{* *}$ & $0.29 * *$ \\
\hline Ejection fraction & $-0.16^{*}$ & -0.08 \\
\hline Mild aortic regurgitation & $0.14^{* * *}$ & $0.29 * *$ \\
\hline Stroke volume & $0.26^{* *}$ & -0.13 \\
\hline Cardiac output & $0.24^{* *}$ & -0.13 \\
\hline Peripheral vascular resistance & $-0.20 * *$ & $0.16 * * *$ \\
\hline
\end{tabular}

${ }^{*} P<0.01 ; * * P<0.001 ; * * * P<0.05$.

Table 5 Regression analyses of the association between selected independent variables and AoR size/dilatation in hypertensive women

Step Variables $R^{2}$ change P-value

Dependent: AoR size (model 1)

$\begin{array}{llll}1 & \text { Cardiac output } & 0.040 & 0.0008 \\ 2 & \text { Waist circumference } & 0.035 & 0.001 \\ 3 & \text { Mild aortic regurgitation } & 0.025 & 0.007 \\ 4 & \text { Age } & 0.017 & 0.02 \\ 5 & \text { Left ventricular mass index } & 0.015 & 0.03 \\ 6 & \text { Height } & 0.013 & 0.04\end{array}$

Dependent: AoR dilatation (model 2)

Hazard ratio

$\begin{array}{llll}1 & \text { Cardiac output }>5.5 \text { I per min } & 5.80 & 0.004 \\ 2 & \text { Mild aortic regurgitation } & 3.87 & 0.02 \\ 3 & \text { Waist circumference }>105 \mathrm{~cm} & 3.46 & 0.03\end{array}$

Abbreviation: AoR, aortic root.

Independent variables in model 1 also included body surface area, diastolic blood pressure, menopause status, homeostasis model assessment index and use of antihypertensive medications. In model 2 age $>60$ years, height $>1.55 \mathrm{~m}$, body surface area $>1.70 \mathrm{~m}^{2}$, diastolic blood pressure $>90 \mathrm{~mm} \mathrm{Hg}$, left ventricular mass index $>80 \mathrm{~g} / \mathrm{h}^{2.7}$, menopause status, homeostasis model assessment index $>3.5$ and use of antihypertensive medications were also included as independent variables. Only variables with significant association are presented.

cardiac output, waist circumference, mild aortic regurgitation, age, LV mass index and height, whereas cardiac output, mild aortic regurgitation and waist circumference were independently related to AoR dilatation in this gender (Table 5). In men, both AoR size and AoR dilatation associated with mild aortic regurgitation, relative wall thickness and $\log \mathrm{C}$-reactive protein (Table 6).

\section{DISCUSSION}

In this report, we evaluated a sample of hypertensive patients with LV hypertrophy and found that (1) AoR size and dilatation were paralleled
Table 6 Regression analyses of the association between selected independent variables and AoR size/dilatation in hypertensive men

\begin{tabular}{llll}
\hline Step & Variable & $R^{2}$ change & P-value \\
\hline Dependent: AoR size (model 1) & & \\
& & & \\
1 & Mild aortic regurgitation & 0.111 & 0.00002 \\
2 & Relative wall thickness & 0.071 & 0.0003 \\
3 & Log C-reactive protein & 0.037 & 0.007
\end{tabular}

Dependent: AoR dilatation (model 2)

Hazard ratio

1 Relative wall thickness $\geqslant 0.45 \quad 11.65 \quad 0.0002$

$2 \quad$ Log C-reactive protein $>-0.41 \mathrm{mg}$ per $100 \mathrm{ml} \quad 6.77 \quad 0.002$

$\begin{array}{llll}3 & \text { Mild aortic regurgitation } & 5.84 & 0.007\end{array}$

Abbreviation: AoR, aortic root.

Independent variables in model 1 also included age, height, body surface area, diastolic blood pressure, left ventricular mass index, peripheral vascular resistance and use of antihypertensive medications. In model 2, age $>60$ years, height $>1.66 \mathrm{~m}$, body surface area $>1.95 \mathrm{~m}^{2}$, diastolic blood pressure $>90 \mathrm{~mm} \mathrm{Hg}$, left ventricular mass index $>80 \mathrm{~g} / \mathrm{h}^{2.7}$, peripheral vascular resistance $>1550$ dynes $\sec \mathrm{cm}^{-5}$ and use of antihypertensive medications were also included as independent variables. Only variables with significant association are presented.

by higher cardiac output and increased waist circumference in women; (2) LV concentric hypertrophy and C-reactive protein were independently associated with AoR size and dilatation in hypertensive men; and (3) although LV mass associated with AoR diameter in both genders, LV end-diastolic diameter and LV wall thickness were actually the foremost correlates of AoR size in women and men, respectively. Overall, these findings suggest that gender-specific mechanisms might be implicated in the regulation of AoR size in hypertensive subjects with LV hypertrophy.

In our study, hypertensive women with AoR dilatation showed increased stroke volume and cardiac output as well as reduced peripheral vascular resistance compared with those with normal AoR. Accordingly, cardiac output emerged as an independent determinant of female AoR size after multivariate analysis. These results point toward a role of volume load in the regulation of AoR diameter in this gender. In agreement with this assumption, larger AoR diameters have been described in individuals with volume overload, such as marathon runners and patients with anemia and aortic valve regurgitation..$^{20-22}$ Moreover, results of the LIFE study showed that stroke volume, a putative marker of volume load, was an independent predictor of AoR diameter, strengthening the notion that proximal aortic structure is sensitive to changes in volume status in hypertensive subjects. ${ }^{5}$ One potential cause of volume overload in hypertensive patients is obesity. ${ }^{23}$ In fact, in our study, AoR size was shown to correlate with body mass index and body surface area in women. Nonetheless, the present findings indicated that abdominal rather than global obesity was more related to AoR size in this gender. In this context, waist circumference came up as the anthropometric measurement showing the strongest correlation with AoR size and was independently associated with AoR diameter. Noticeably, the relationship between waist circumference and AoR size was independent of the homeostasis model assessment index, suggesting that insulin resistance had no major role in this regard. These results are in agreement with a recent report from our group showing that waist circumference was associated with the LV enddiastolic diameter independently of insulin resistance and body mass index in hypertensive women with a high prevalence of LV hypertrophy. ${ }^{10}$ Altogether, these data seem to suggest that circulating volume derived from central adiposity might be involved in AoR and LV chamber enlargement in hypertensive women. Conversely, although the relationship between AoR size and cardiac output and central 
obesity was not influenced by menopause status, we cannot rule out the role of estrogens in this regard.

In contrast to the results obtained from females, LV concentric hypertrophy showed a major relationship with AoR size in males. Similarly, the positive association between LV mass and AoR size was mainly dependent on LV wall thickness in men, whereas it was mostly reliant on LV chamber diameter in women. These findings might provide some clues regarding the regulatory mechanisms of male AoR size. First, they indicate that growth factors influencing the myocardium may be also involved in AoR remodeling. Second, they suggest that pressure overload might be a potential hemodynamic stimulus for AoR dilatation, given that hypertension is strongly related to $\mathrm{LV}$ concentricity. ${ }^{24}$ Traditionally, chronic exposure to high intra-arterial pressures in hypertension is believed to accelerate elastin breakdown, and is therefore believed to further promote proximal aortic dilatation. ${ }^{25}$ However, this assumption is challenged by the absence of a significant relationship between blood pressure and AoR size in our studied sample. Although the use of antihypertensive medications and evaluation of clinic blood pressure levels might be major limitations to this assumption, our data agree with other studies showing no independent impact of clinic blood pressure measurements on AoR size either in treated or untreated hypertensives. ${ }^{4-6}$ Conversely, a recent report showed a direct significant association between nighttime blood pressure evaluated by ambulatory blood pressure monitoring and AoR diameter in hypertensive patients, ${ }^{7}$ supporting the notion that pressure overload might indeed influence the AoR phenotype.

Although AoR dilatation may take place as a result of cardiac and hemodynamic factors, reverse causality may also occur. For instance, mild aortic regurgitation secondary to AoR dilatation could also influence cardiac output and cardiac remodeling. ${ }^{22}$ In this context, our data from multivariate analysis showed that mild aortic regurgitation was indeed associated with AoR size/dilatation in both genders. Nevertheless, this variable did not explain the relationship between AoR dilatation and cardiac output in women and exerted no influence on the association between AoR dilatation and relative wall thickness in men, indicating that the cardiac and hemodynamic factors were related to AoR enlargement independently of aortic regurgitation. However, it must be acknowledged that the cross-sectional nature of our study does not exclude the possibility that the systemic hemodynamic parameters and LV remodeling were influenced by hemodynamic abnormalities secondary to the increased AoR size.

A notable result of our study was that men showed no variation in body size according to AoR diameter. This finding argues against data obtained from population-based and untreated hypertensives showing a direct impact of body surface area on AoR size. ${ }^{4-7}$ Nonetheless, they seem to agree with the results obtained from patients with electrocardiographic LV hypertrophy, which showed no difference in the body surface areas of patients with either enlarged or normal AoR diameter. ${ }^{5}$ It is noteworthy that most of the subjects with dilated AoR described in this aforementioned study were of male gender, thereby supporting the notion that body size may not be a major determinant of AoR dilatation in hypertensive hypertrophic men.

Left ventricular hypertrophy and carotid intima-media thickness have been independently related to microalbuminuria and retinal vascular changes in hypertensive subjects. ${ }^{26-28}$ These observations raise the assumption that hypertension-induced cardiac and macrovascular damages share common pathophysiological mechanisms with microvascular disease. In this context, in this study, we evaluated whether there was a relationship between AoR size and markers of microvascular injury. Noticeably, we reproduced data from other sources showing no association between AoR size and microalbuminuria. ${ }^{6,7}$ Furthermore, we found that AoR size did not correlate with creatinine clearance and retinal vascular changes in both genders, thus suggesting that, in contrast to LV hypertrophy and carotid intimamedia growth, AoR dilatation might not show an expressive relationship with hypertensive microvascular damage.

A potential limitation of this study is that the majority of hypertensive patients were on medications. Some findings might therefore be attributable to the differential effect of various therapy regimens. However, this hypothesis seems less probable as the univariate correlations showed no significant relationship between antihypertensive medications and AoR size in both genders. Moreover, we diminished this potential bias by considering the presence of antihypertensive treatment in multivariate models.

In conclusion, the present findings indicate that there are sexspecific determinants of AoR size in hypertensive patients with LV hypertrophy. In this regard, our results suggest that volume overload and central obesity are related to AoR dilatation in women, whereas AoR enlargement is associated more with LV myocardial growth and inflammatory status in men. Nevertheless, further longitudinal studies are required to confirm these assumptions.

\section{ACKNOWLEDGEMENTS}

This study was sponsored by grants from Fundação de Amparo à Pesquisa do Estado de São Paulo (Proc. 05/56986-5) and Conselho Nacional de Desenvolvimento Científico e Tecnológico (Proc. 304329/06-1 and 474206/07-6).

1 Seder JD, Burke JF, Pauletto FJ. Prevalence of aortic regurgitation by color flow Doppler in relation to aortic root size. J Am Soc Echocardiogr 1990; 3: 316-319.

2 Gardin JM, Arnold AM, Polak J, Jackson S, Smith V, Gottdiener J. Usefulness of aortic root dimension in persons $>$ or 65 years of age in predicting heart failure, stroke, cardiovascular mortality, all-cause mortality and acute myocardial infarction (from the Cardiovascular Health Study). Am J Cardiol 2006; 97: 270-275.

3 Kim M, Roman MJ, Cavallini MC, Schwartz JE, Pickering TG, Devereux RB. Effect of hypertension on aortic root size prevalence of aortic regurgitation. Hypertension 1996; 28: 47-52.

4 Palmieri V, Bella JN, Arnett DK, Roman MJ, Oberman A, Kitzman DW, Hopkins PN, Paranicas M, Rao DC, Devereux RB. Aortic root dilatation at sinuses of valsalva aortic regurgitation in hypertensive normotensive subjects. The hypertension genetic epidemiology network study. Hypertension 2001; 37: 1229-1235.

5 Bella JN, Wachtell K, Boman K, Palmieri V, Papademetriou V, Gerdts E, Aalto T, Olsen $\mathrm{MH}$, Olofsson M, Dahlöf B, Roman MJ, Devereux RB. Relation of left ventricular geometry function to aortic root dilatation in patients with systemic hypertension left ventricular hypertrophy (the LIFE Study). Am J Cardiol 2002; 89: 337-341.

6 Cuspidi C, Meani S, Fusi V, Valerio C, Sala C, Zanchetti A. Prevalence correlates of aortic root dilatation in patients with essential hypertension: relationship with cardiac extracardiac target organ damage. J Hypertens 2006; 24: 573-580.

7 Cuspidi C, Meani S, Valerio C, Esposito A, Sala C, Maisaidi M, Zanchetti A, Mancia G. Ambulatory blood pressure target organ damage aortic root size in never treated essential hypertensive patients. J Hum Hypertens 2007; 21: 531-538.

8 Farasat SM, Morrell CH, Scuteri A, Ting CT, C P Yin F, Spurgeon HA, Chen CH, G Lakatta E, Najjar SS. Do hypertensive individuals have enlarged aortic root diameters? Insights from studying the various subtypes of hypertension. Am J Hypertens 2008; 21: 558-563.

9 de Simone G, Devereux RB, Roman MJ, Alderman MH, Laragh JH. Relation of obesity gender to left ventricular hypertrophy in normotensive hypertensive adults. Hypertension 1994; 23: 600-606.

10 Pio-Magalhães JA, Cornélio M, Leme Jr CA, Matos-Souza JR, Garlipp CR, Gallani MC, Rodrigues RC, Franchini KG, Nadruz Jr W. Upper arm circumference is an independent predictor of left ventricular concentric hypertrophy in hypertensive women. Hypertens Res 2008; 31: 1177-1183.

11 Shigematsu Y, Norimatsu S, Ohtsuka T, Okayama H, Higaki J. Sex-related differences in the relations of insulin resistance obesity to left ventricular hypertrophy in Japanese hypertensive patients. Hypertens Res 2006; 29: 499-504.

12 Saitoh M, Nishimura H, Tanaka T, Kondoh T. Gender-related differences in target organ damage in untreated patients with essential hypertension. Intern Med 2006; 45: 377-383.

13 Executive Summary: The Third Report of The National Cholesterol Education Program (NCEP) Expert Panel on Detection, Evaluation And Treatment of High Blood Cholesterol in Adults (Adult Treatment Panel III). JAMA 2001; 285: 2486-2497. 
14 Sales ML, Ferreira MC, Leme Jr CA, Velloso LA, Gallani MC, Colombo RC, Franchini KG, Nadruz Jr W. Non-effect of p22-phox -930A/G polymorphism on end-organ damage in Brazilian hypertensive patients. J Hum Hypertens 2007; 21: 504-506.

15 Lang RM, Bierig M, Devereux RB, Flachskampf FA, Foster E, Pellikka PA, Picard MH, Roman MJ, Seward J, Shanewise J, Solomon S, Spencer KT, St John Sutton M, Stewart W. Recommendations for chamber quantification. Eur J Echocardiogr 2006; 7: 79-108.

16 Kontos J, Papademetriou V, Wachtell K, Palmieri V, Liu JE, Gerdts E, Boman K, Nieminen MS, Dahhlöf $B$, Devereux RB. Impact of valvular regurgitation on left ventricular geometry function in hypertensive patients with left ventricular hypertrophy: the LIFE study. J Hum Hypertens 2004; 16: 431-436.

17 Dubin J, Wallerson DC, Cody RJ, Devereux RB. Comparative accuracy of Doppler echocardiographic methods for clinical stroke volume determination. Am Heart J 1990; 120: $116-123$.

18 Lauer MS, Anderson KM, Larson MG, Levy D. A new method for indexing left ventricular mass for differences in body size. Am J Cardiol 1994; 74: 487-491.

19 Savage DD, Garrison RJ, Kannel WB, Levy D, Anderson SJ, Stokes III J, Feinleib M, Castelli WP. The spectrum of left ventricular hypertrophy in a general population sample: the Framingham Study. Circulation 1987; 75: I26-133.

20 Zoneraich S, Rhee JJ, Zoneraich O, Jordan D, Appel J. Assessment of cardiac function in marathon runners by graphic noninvasive techniques. Ann N Y Acad Sci 1977; 301: 900-917.

21 Lester LA, Sodt PC, Hutcheon N, Arcilla RA. Cardiac abnormalities in children with sickle cell anemia. Chest 1990; 98: 1169-1174.
22 Padial LR, Oliver A, Sagie A, Weyman AE, King ME, Levine RA. Two-dimensional echocardiographic assessment of the progression of aortic root size in 127 patients with chronic aortic regurgitation: role of the supraaortic ridge and relation to the progression of the lesion. Am Heart J 1997; 134: 814-821.

23 Dreslinski GR, Ventura HO, Oigman W, Frohlich ED, Dunn FG. Dimorphic cardiac adaptation to obesity arterial hypertension. Ann Intern Med 1983; 99: 757-761.

24 Verdecchia P, Angeli F, Achilli P, Castellani C, Broccatelli A, Gattobigio R, Cavallini C. Echocardiographic left ventricular hypertrophy in hypertension: marker for future events or mediator of events? Curr Opin Cardiol 2007; 22: 329-334.

25 O'Rourke MF, Nichols WW. Aortic diameter aortic stiffness wave reflection increase with age isolated systolic hypertension. Hypertension 2005; 45: 652-658.

26 Tikellis G, Arnett DK, Skelton TN, Taylor HW, Klein R, Couper DJ, Richey Sharrett A, Yin Wong T. Retinal arteriolar narrowing left ventricular hypertrophy in African Americans. the Atherosclerosis Risk in Communities (ARIC) study. Am J Hypertens 2008; 21: 352-359.

27 Pontremoli R, Ravera M, Bezante GP, Viazzi F, Nicolella C, Berruti V, Leoncini G, Del Sette M, Brunelli C, Tomolillo C, Deferrari G. Left ventricular geometry function in patients with essential hypertension microalbuminuria. J Hypertens 1999; 17: 993-1000.

28 Takiuchi S, Kamide K, Miwa Y, Tomiyama M, Yoshii M, Matayoshi T, Horio T, Kawano Y. Diagnostic value of carotid intima-media thickness plaque score for predicting target organ damage in patients with essential hypertension. J Hum Hypertens 2004; 18 : 17-23.

Supplementary Information accompanies the paper on Hypertension Research website (http://www.nature.com/hr) 